Journal of Animal and Veterinary Advances 9 (15): 2074-2081, 2010

ISSN: $1680-5593$

(C) Medwell Journals, 2010

\title{
Spatio-Temporal Expression of Matrix Metalloproteinases-2 and -9 in Porcine Endometrium During Implantation
}

\author{
Qian Ren, Shu Guan, Jinluan Fu and Aiguo Wang \\ College of Animal Science and Technology, China Agricultural University, \\ Beijing, People's Republic of China
}

\begin{abstract}
Implantation is a complex process and involves various molecules to maintain endometrial function. The remodeling of endometrial matrices is a necessary process during implantation. Matrix-Metalloproteinases (MMPs) like gelatinases (MMP-2 and -9) are considered to play important roles in this process. Real-time PCR, western blot and immunostaining analysis were used to study MMP-2 and -9 expression between/at attachment sites of endometrium of Days 13,18 and 24 pregnant sows. The results indicate that MMP- 2 mRNA and protein expression is higher than that of MMP-9 during implantation so it was proposed that MMP-2 plays a more vital role in endometrium during implantation in sows. During implantation, MMP-2 protein expression at attachment sites was higher than between attachment sites and this effect was significant at days $18(\mathrm{p}<0.01)$ and 24 $(\mathrm{p}<0.01$ ) of pregnancy. The immunostaining of MMP-2 and -9 was found in stroma and luminal epithelium and the immunostaining of MMP-2 in stroma at attachment sites was stronger than between attachment sites. These results suggest that MMP-2 and -9 may mediate ECM degradation and remodeling of endometrium at attachment sites is greater than between attachment sites. The immunostaining of MMP-2 and -9 in stroma indicate the endometrial stromal decidualization-like in sows.
\end{abstract}

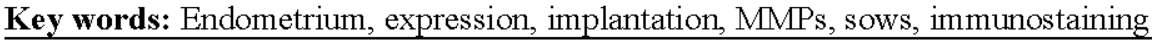

\section{INTRODUCTION}

Implantation is the process in which mammalian embryos attach to maternal uterus and interact intimately to form a placenta. In rodents and primates, implantation is highly invasive and characterized by deep penetration of the endometrium by the implanting embryo. In contrast, porcine embryos attachment to the uterine wall is noninvasive and superficial. Porcine embryos begin to attach to the uterus on day 13 of pregnancy with attachment complete between days 18 and 24 (Kyriazakis and Whittemore, 2006). Successful implantation is the result of reciprocal interactions between the implantationcompetent blastocyst and receptive uterus.

During implantation, degradation and regeneration of the endometrial Extra-Cellular Matrix (ECM) is a vital process which involves Matrix Metalloproteinases (MMPs). MMPS, including MMP-2 (gelatinase A) and MMP-9 (gelatinase B) play an important role in tissue remodeling in various physiological and pathological processes such as implantation, ovarian and uterine functions during peri-partum, wound healing, cancer development etc. (Nagase and Woessner, 1999; Takagi et al., 2007). The roles of MMPs during implantation in humans and rodents (invasive placentation) are known (Wooding 1992; Bai et al., 2005; Rechtman et al., 1999; Xu et al., 2000; Das et al., 1997; Huppertz et al., 1998; Bjorn et al., 2000) but knowledge about endometrium remodeling in ungulates (noninvasive placentation) is limited. In sheep, the important roles of MMPs in endometrial remodeling, especially those of MMP-1 and -2 were reported (Salamonsen, 1999). MMP-2 activity is regulated by co-localized membranetype 1 MMP (MT1-MMP) and tissue inhibitor of metalloproteinase-2 (TIMP-2) and they control endometrium remodeling during gestation in goats (Uekita et al., 2004).

Porcine embryos undergo true epitheliochorial placentation in which luminal epithelium remains morphologically intact and the embryos trophectoderm simply attaches to the apical luminal epithelium surface without displacement or invasion of uterine stromal cells (Burghardt et al., 2002). The detailed expression profiles of gelatinases have not been clarified in Porcine endometrium during implantation; namely, the proteolysis mechanisms of the endometrial ECM are still obscure during implantation in sows. During implantation, trophoblast cells eliminate luminal epithelial cells and the

Corresponding Author: Jinluan $\mathrm{Fu}$, College of Animal Science and Technology, China Agricultural University, Beijing, People's Republic of China 
epithelium is reorganized (Yamada et al., 2002a, b. Gelatinases may play a significant role in this process. During implantation, the newly formed binucleate and multinucleate cells produce many molecules, peptides and hormones such as placental lactogen, pregnancyassociated glycoproteins, prolactin-related proteins, steroid hormones and heparanase (Kizaki et al., 2003; Wooding, 1992; Yamada et al., 2002a, b; Patel et al., 2004). Compared to invasive placentation, the mechanisms of this type of proteinase is unknown in sows but it is just as important. Most embryo loss occurs during implantation in sows. The cause of this usually relates to the uterine circumstances which depend on humoral regulation and may depend on the spacio-temporal condition of the endometrium. Therefore, will detect MMP-2 and -9 spatiotemporal expression in porcine endometrium during implantation to investigate the function of MMP-2 and -9 .

\section{MATERIALS AND METHODS}

\section{Animals and tissue collection}

Animals: Multiparous Yorkshire sows (5th parity) were observed daily for estrous behavior in the presence of a boar. Sows exhibiting at least two estrous cycles of normal duration (21 days) were inseminated twice, 12 and $24 \mathrm{~h}$ after estrus detection. Fifteen sows were slaughtered ( $\mathrm{n}=5 /$ day) by electrical stunning on days 13,18 and 24 of pregnancy. The day of $24 \mathrm{~h}$ after estrus detection was considered Day 0 . The slaughter was conducted according to procedure of Animal Welfare Committee in China Agricultural University.

Tissue collection: Endometrial tissue sampling was carried out according to the procedure of Lord with minor modifications (Lord et al., 2006). Prior to endometrial tissue collection, the uteri of day 13 pregnant sows were flushed with PBS and pregnancy confirmed by the presence of embryos in the flushing. The reproductive tract was collected and the uterine horns were opened along the antimesometrial border. Endometrial tissue samples were taken from the mesometrial side, at the sites or between sites of conceptus attachment for the pregnant sows or in the middle of the horn for the cyclic sows. Attachment sites from day 13 sows were determined by the presence of local hyperemia in endometrial tissue, manifest as a darker reddish color compared to surrounding tissue. Several sections of each uterine horn of sows from each state were collected immediately. For immunohistochemistry, specimens $\left(1.5 \mathrm{~cm}^{3}\right)$ were fixed in $4 \%(\mathrm{w} / \mathrm{v})$ paraformaldehyde in PBS $(\mathrm{pH} 7.4)$ at $4^{\circ} \mathrm{C}$ overnight, paraffin embedded, sectioned and stained with haematoxylin-eosin. For RNA extraction, specimens were placed in RNAlater (Qiagen, Valencia, CA, USA) at $4^{\circ} \mathrm{C}$ overnight and then stored at $-20^{\circ} \mathrm{C}$. For Western blot analysis, the samples were stored at $-80^{\circ} \mathrm{C}$ until processed.

RNA extraction and cDNA synthesis: Trizol reagent (InVitrogen, Carlsbad, CA, USA) was used to extract total RNA according to the manufacturer's instructions. RNA was kept at $-80^{\circ} \mathrm{C}$ until used. The purity and integrity of RNA was electrophoretically tested by ethidium bromide staining, Optical Density (OD) absorption ratio $\mathrm{OD}_{260} / \mathrm{OD}_{280}(>1.90)$ and $\mathrm{rRNA}(28 / 18 \mathrm{sec})$ ratios (=2), respectively. Two micrograms of total RNA were reversetranscribed into cDNA in the presence of polythymidine oligonucleotide primers (Oligo-dT18) and Moloney Murine Leukemia Virus Reverse Transcriptase (MMLVRT; Promega, Madison, WI, USA) in a total reaction volume of $25 \mu \mathrm{L}$. RT products were stored at $-20^{\circ} \mathrm{C}$ for use.

Primer design: The mRNA sequences of porcine MMP-2 (GenBank NM214192) and MMP-9 (GenBank DQ132879) were used to design two pairs of primers (Table 1). To ensure amplification of only the Complementary DNA (cDNA) but not the Genomic DNA (gDNA), the forward and reverse primers used for amplification were placed in two different exons of the gene and they were all directly against the highly conserved region of sequence. Primers were designed using Primer express software v. 3.0 (Applied Biosystems, Foster City, CA, USA). Housekeeping gene GAPDH was assayed as normalization control to correct for loading discrepancies for all samples assayed. Primer for GAPDH was used according to Lin et al. (2007). They were shown in Table 1. Primers were synthesized by Shanghai Sangon Biological Engineering Technology And Service Co., Ltd (Shanghai, China).

Real-time Polymerase Chain Reaction (RT-PCR): RTPCR was performed with an ABI Prism 7900HT (Applied Biosystems, Foster City, CA, USA) Sequence Detection System using SYBR green PCR master mix (Applied Biosystems) to analyze MMP-2 and -9 expression in endometrium of sows (days 13, 18 and 24 of pregnancy). Reactions were prepared in $25 \mu \mathrm{L}$ volume consisting of $1.5 \mu \mathrm{L}$ RT product, $0.5 \mu \mathrm{L}$ forward and reverse primers each $\left(10\right.$ pmol $\left.\mu \mathrm{L}^{-1}\right), 12.5 \mu \mathrm{L}$ SYBR green PCR master mix and $10 \mu \mathrm{L}$ double distilled water. PCR thermal cycling conditions were $50^{\circ} \mathrm{C}$ for $2 \mathrm{~min}$ and $95^{\circ} \mathrm{C}$ for $10 \mathrm{~min}$, followed by 40 cycles of $95^{\circ} \mathrm{C}$ for $15 \mathrm{sec}$ and $60^{\circ} \mathrm{C}$ for $30 \mathrm{sec}$. After each PCR reaction, melting curves were obtained by stepwise increases in the temperature from 60 to $95^{\circ} \mathrm{C}$ to ensure single product amplification. In PCR 
Table 1: Oligonucleotide primers used for Real-Time PCR (RT-PCR) of Porcine MMP-2, -9 and a house keeping gene

\begin{tabular}{llccc}
\hline Primer name & Primer sequences $\left(5^{\prime}-3^{\prime}\right)$ & Annealing temperature $\left({ }^{\circ} \mathrm{C}\right)$ & Product size $(\mathrm{bp})$ & GenBank accession No./references \\
\hline MMP-2 & Forward:CGCCCATCATCAAGTTTC & 60 & 175 & NM214192 \\
MMP-9 & $\begin{array}{l}\text { Reverse: TCGAGTTCGCCTGTCTG } \\
\text { Forward:TGAAGACGCAGAAGGTGGATTC }\end{array}$ & 60 & 140 & DQ132879 \\
GAPDH & $\begin{array}{l}\text { Reverse:GGCCTGGCAGAAGTAAGCTTC } \\
\text { Forward:GTCCACTGGTGTCTTCACGA } \\
\text { Reverse:GCTGACGATCTTGAGGGAGT }\end{array}$ & 60 & 154 & AF141959/Lin et al., 2007 \\
\hline
\end{tabular}

reactions, RNA and gDNA were used as negative and positive controls, respectively and no amplicons were obtained by using RNA directly. All samples were measured in triplicates. The identity of PCR products were verified by sequence analysis after cloning into the $\mathrm{pMD}$ 18-T vector (TaKaRa, Dalian, China). Relative abundance of MMP-2 and -9 mRNA normalized to GAPDH was analyzed by $2^{-\Delta c t}$ comparative $\mathrm{C}_{\mathrm{t}}$ method (Livak and Schmittgen, 2001; Schmittgen and Livak, 2008).

Western blot: Protein fractions were obtained using a procedure described earlier by Huang et al. (2006) with minor modifications. Endometrial tissues (days 13, 18 and 24 of pregnancy) were homogenized in fresh lysis buffer containing $1 \%(\mathrm{v} / \mathrm{v})$ Triton X-100, $20 \mathrm{mM}$ Tris- $\mathrm{HCl}(\mathrm{pH}$ 8.0), $160 \mathrm{mM} \mathrm{NaCl}, 1 \mathrm{mM} \mathrm{CaCl}_{2}$ and $1 \mathrm{mMPMSF}$ followed by ultrasonication (three 5 -sec bursts). After centrifugation $\left(10,000 \mathrm{~g}, 15 \mathrm{~min}, 4^{\circ} \mathrm{C}\right)$, insoluble materials were removed and supernatants were stored at $-80^{\circ} \mathrm{C}$ for further analysis.

Fifty micrograms of total proteins were dissolved in SDS gel-loading buffer containing $50 \mathrm{mM}$ Tris- $\mathrm{HCl}$ ( $\mathrm{pH}$ 6.8), 4\% SDS, $20 \%$ glycerol and $2 \% \beta$-mercaptoethanol, heated to $95^{\circ} \mathrm{C}$ for $4 \mathrm{~min}$ and separated on $10 \%$ SDSPAGE. Separated proteins were electroblotted onto $0.2 \mu \mathrm{m}$ nitrocellulose membrane in transfer buffer containing 20mMTris- $\mathrm{HCl}$ buffer ( $\mathrm{pH} 8.2$ ), $150 \mathrm{mM}$ glycine, $20 \%$ methanol, $0.05 \% \mathrm{SDS}$. The nonspecific binding sites were blocked with $5 \%$ nonfat dry milk in TBS-T buffer for $1.5 \mathrm{~h}$ at room temperature.

After blocking, the nitrocellulose membrane was incubated overnight at $4^{\circ} \mathrm{C}$ with a mouse monoclonal antibody against human (MMP-2: MAB3308, CHEMICON, diluted 1:500; MMP-9: SC-21733, Santa Cruz, diluted 1:200) and a goat polyclonal antibody anti-human ACTB (sc-1616, Santa Cruz, Califonia, CA, USA) diluted 1:1000. ACTB was an internal control. Subsequently, membrane was incubated with anti-mouse HRP conjugated secondary antibodies (Zymed) for $2 \mathrm{~h}$ at room temperature. Immune complexes were visualized with an ECL detecyion reagent on ECL Hyperfilm (both Amersham, England). The MMP-2 and -9 signals were quantified and normalized to ACTB signals by the use of Quantity One software (Bio-Rad, Hercules, CA, USA).
Immunohistochemistry: Immunohistochemistry was performed by the labeled streptavidin/peroxidase biotin method (Zymed, South San Francisco, CA, USA) to analyze MMP-2 and -9 expression in endometrium of sows (days 13, 18 and 24 of pregnancy). The tissue sections were cut at $4 \mu \mathrm{m}$ thickness and mounted on silanized slides, dewaxed in xylene and rehydrated in graded ethanol. Sections were treated with $3 \% \mathrm{H}_{2} \mathrm{O}_{2}$ in PBS for 10 min to quench endogenous peroxidase activity. Sections were then incubated in 5\% goat serum in PBS for $20 \mathrm{~min}$ to reduce nonspecific binding.

After tapping the excess goat serum solution, sections were incubated overnight at $4^{\circ} \mathrm{C}$ with the same MMP- $2^{\circ} \mathrm{C}$ diluted $1: 1000$ ) and $\mathrm{MMP}-9^{\circ} \mathrm{C}$ diluted $1: 100$ ) antibodides applied in western blotting, then incubated for $20 \mathrm{~min}$ in biotinylated goat anti-mouse antibody (Zymed) followed by incubation with HRP-streptavidin (Zymed) for $20 \mathrm{~min}$. The antibody binding sites were visualized by incubating the tissue sections with $D A B$ solution provided by a DAB kit (Zymed). Finally, sections were counterstained with haematoxylin, dehydrated and mounted. For the negative controls mouse antibody (Zymed) was used at the same concentration as primary antibodies. Images of the sections were captured using Olympus microscope BX51 and digital camera DP70 (Olympus, Tokyo, Japan).

Statistical analysis: The data were expressed as means \pm SEM. The statistical comparison of relative $\mathrm{mRNA}$ and protein expression of MMP-2 and -9 between experimental groups were analyzed by all pair-wise multiple comparison procedures (Tukey test) or by a twoway ANOVA (full factorial on sampling site and day of pregnancy) where pertinent. Data were analyzed using SAS 8.02 Software. Statistical significance was determined at $\mathrm{p}<0.05$.

\section{RESULTS AND DISCUSSION}

Expression of MMP-2 and -9 mRNA in porcine endometrium: The effect of the day of pregnancy on MMP-2 mRNA expression in the porcine endometrium during the embryo implantation was shown in Fig. 1a. The expression in pregnant sows was highest by day 13 compared with days $18(\mathrm{p}<0.01)$ and $24(\mathrm{p}<0.01)$; There 


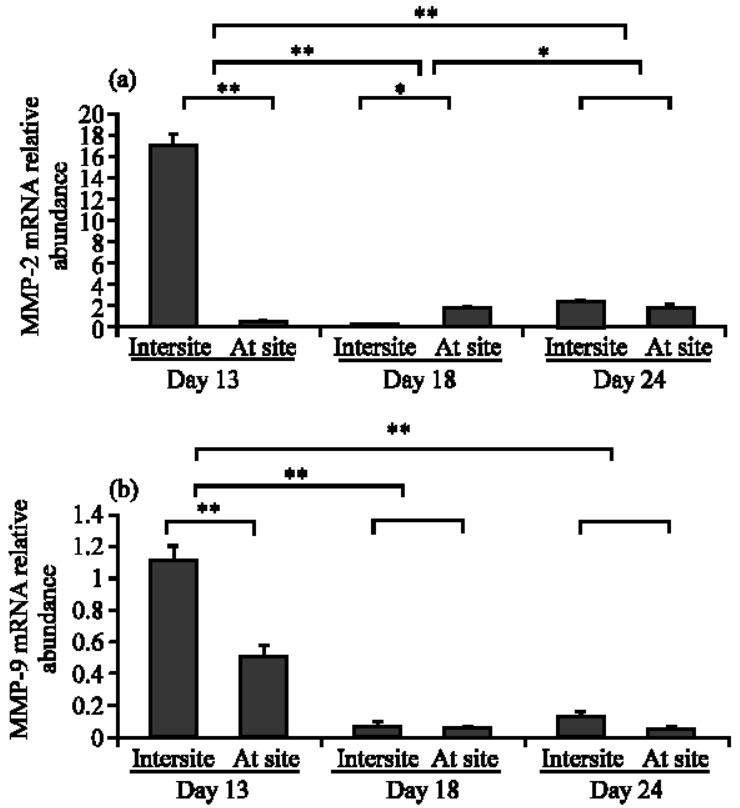

Fig. 1: Effects of the day of pregnancy and site of endometrial tissue sampling on relative expression of MMP-2 and -9 mRNA in endometrial tissue. Data are ratios of MMP-2 and -9 relative mRNA abundance normalized to GAPDH.. Each bar represents means \pm SEM; ${ }^{*} \mathrm{p}<0.05, \quad * * \mathrm{p}<0.01$. Intersite $=$ between attachment site; At site $=$ at attachment site

were significant differences between days 18 and 24 pregnant sows $(p<0.05)$. The effect of the site of endometrial tissue sampling on MMP- 2 mRNA expression during the embryo implantation was shown in Fig. 1a. The expression was higher between attachment sites compared with at attachment sites at days 13 and 24 of pregnancy and this effect was significant at day 13 ( $<<0.01$ ); In contrast, The expression was lower between attachment sites compared with at attachment sites at day 18 of pregnancy and this effect was significant $(\mathrm{p}<0.05)$. The effect of the day of pregnancy on MMP-9 mRNA expression in the porcine endometrium during the embryo implantation was shown in Fig. 1b. The expression in pregnant sows was highest by day 13 compared with days $18(p<0.01)$ and $24(p<0.01)$. The effect of the site of endometrial tissue sampling on MMP-9 mRNA expression during the embryo implantation was shown in Fig. 1b. The expression was higher between attachment sites compared with at attachment sites and this effect was significant at day13 $(\mathrm{p}<0.01)$.

Expression of MMP-2 and $\mathbf{- 9}$ protein in porcine endometrium: The effect of the day of pregnancy on MMP-2 protein expression in the porcine endometrium during the embryo implantation was shown in Fig. 2a, b. The expression in pregnant sows was highest by day 18 compared with days $13(\mathrm{p}<0.01)$ and $24(\mathrm{p}<0.01)$; There were no significant differences between days 13 and 24 pregnant sows. The effect of the site of endometrial tissue sampling on MMP-2 protein expression in endometrium was shown in Fig. 2b. The expression was lower between attachment sites compared with at attachment sites and this effect was significant at days $18(\mathrm{p}<0.01)$ and 24 $(\mathrm{p}<0.01)$ of pregnancy.

The effect of the day of pregnancy on MMP-9 protein expression in the porcine endometrium during the embryo implantation was shown in Fig. 2c. The expression in pregnant sows was lowest by day 24 compared with days $13(\mathrm{p}<0.01)$ and $18(\mathrm{p}<0.01)$; There were no significant differences between days 13 and 18 pregnant sows. The effect of the site of endometrial tissue sampling on MMP-9 protein expression in endometrium was shown in Fig. 2c. The expression was lower between attachment sites compared with at attachment sites at days 13 and 18 of pregnancy and this effect was significant at day 18 $(\mathrm{p}<0.01)$; In contrast, the expression was higher between attachment sites compared with at attachment sites at day 24 of pregnancy and this effect was significant $(\mathrm{p}<0.01)$.

Localization of MMP-2 and $\mathbf{- 9}$ protein in porcine endometrium: MMP-2 immunostaining was mainly observed in luminal epithelium, glandular epithelium and subepithelial stroma. On day 13 of pregnancy, MMP-2 staining between attachment sites was strong in subepithelial stroma and moderate staining in luminal and glandular epithelium (Fig. 3a and b). At attachment sites, staining was strong in luminal epithelium and moderate staining in stroma and glandular epithelium (Fig. 3c and d). On day 18 of pregnancy, very strong staining were observed in luminal epithelium and strong staining in stroma but weak in glandular epithelium were detected between attachment sites (Fig. 3e and f). At attachment sites staining was very strong in stroma and strong in luminal and glandular epithelium (Fig. $3 g$ and h).

On day 24 of pregnancy staining was moderate in luminal epithelium and weak in stroma and glandular epithelium were detected between attachment sites (Fig. 3i and j). At attachment sites, staining was strong in glandular epithelium and weak in stroma but absent in luminal epithelium (Fig. 3k and 1). A minimal background, but no staining was seen in the negative controls (Fig. $3 \mathrm{~m}$ and n). MMP-9 immunostaining was mainly observed in luminal epithelium, glandular epithelium and subepithelial stroma. On day 13 of pregnancy, MMP-9 staining between attachment sites was strong in subepithelial 


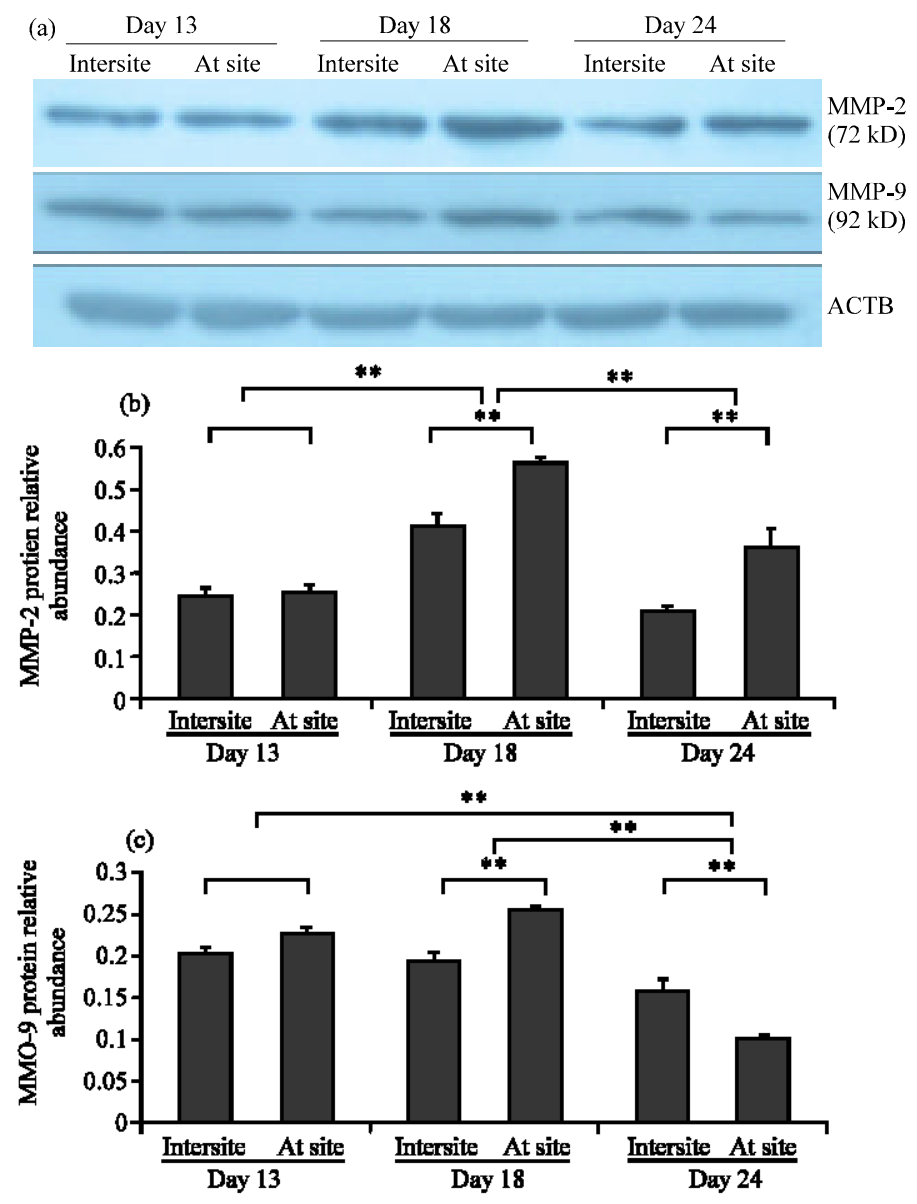

Fig. 2: Effects of the day of pregnancy and site of endometrial tissue sampling on relative expression of MMP-2 and -9 protein in endometrial tissue. a) Western blot of MMP-2 and -9 in endometrial tissue. Molecular weight marker was shown on the right. ACTB was used as an internal control. b) and c) Relative abundance of MMP-2 and -9 protein normalized to ACTB. Each bar represents means \pm SEM; * * $<0.01$. Intersite $=$ between attachment site; At site $=$ at attachment site

stroma and moderate staining in glandular epithelium but weak in luminal epithelium (Fig. 4a and b). At attachment sites, staining was strong in luminal epithelium and strima and moderate staining in glandular epithelium (Fig. 4c and d). On day 18 of pregnancy, strong staining were observed in luminal epithelium and moderate staining in stroma and glandular epithelium were detected between attachment sites (Fig. 4e and f). At attachment sites, staining was very strong in luminal epithelium and stroma and strong in glandular epithelium (Fig. $4 \mathrm{~g}$ and $\mathrm{h}$ ). On day 24 of pregnancy, staining was moderate in luminal epithelium and stroma but weak in glandular epithelium were detected between attachment sites (Fig. $4 i$ and j). At attachment sites, staining was weak in stroma and glandular epithelium but absent in luminal epithelium (Fig. 4k and 1). A minimal background, but no staining, was seen in the negative controls (Fig. $4 \mathrm{~m}$ and $\mathrm{n}$ ).
In this study, MMP-2 and -9 mRNA and protein expression has demonstrated between/at attachment sites of porcine endometrium during different stages of implantation. MMP-2 and -9 abundance varied with the day of pregnancy and the site of endometrial tissue sampling which implied the important role of these genes in implantation of sows. RT-PCR and western-blot revealed that the level of endometrial MMP-2 expression is greater than that of MMP-9, it was proposed that MMP-2 plays a more vital role in endometrium during implantation in sows. It is similar to cows (Kizaki et al., 2008). The results of RT-PCR and western-blot analysis did not completely match which implies that the regulation of MMP-2 and -9 expression can occur at the translational level. Endometrial remodeling is essential for successful implantation and MMPs have been shown to regulate this process in various species (Blankenship and King, 1994; 


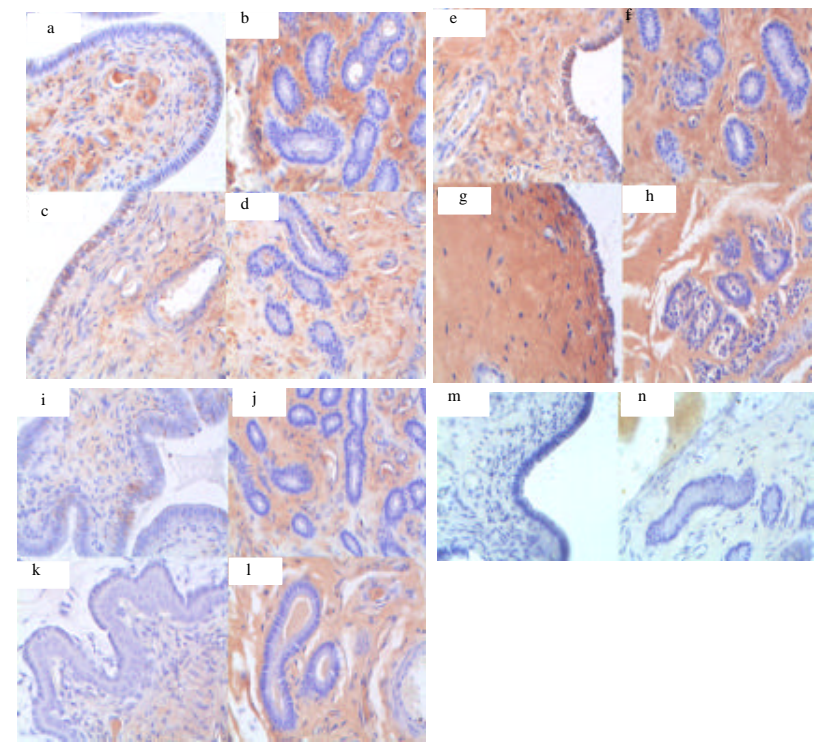

Fig. 3: Immunohistochemical localization of MMP-2 in pig uterus. ( $a$ and $b$ ): Tissue from between attachment sites of day 13 pregnant sows, (c and d): At attachment sites of a day 13 pregnant sows, (e and f): Between attachment sites of a day 18 pregnant sow, ( $g$ and h): At attachment sites of a day 18 pregnant sow, (i and j): Between attachment sites of a day 24 pregnant sow, ( $\mathrm{k}$ and 1): At attachment sites of a day 24 pregnant sow and ( $m$ and $n$ ): Negative controls for localization $(\mathrm{x} 400)$

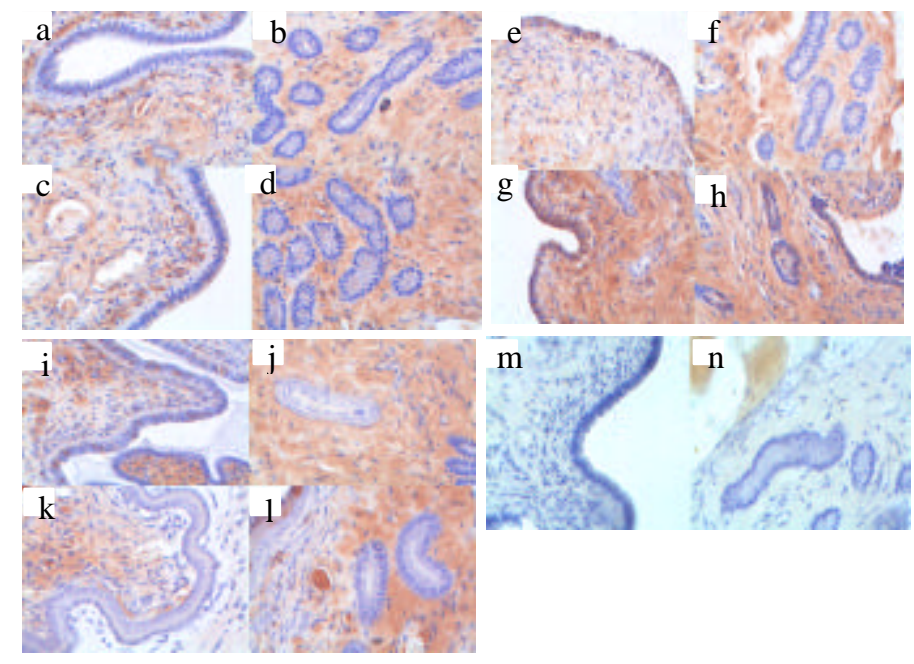

Fig. 4: Immunohistochemical localization of MMP-9 in pig uterus. ( $a$ and $b$ ): Tissue from between attachment sites of day 13 pregnant sows, (c and d): At attachment sites of a day 13 pregnant sows.,(e and f): Between attachment sites of adDay 18 pregnant sow, (g and h): At attachment sites of a day 18 pregnant sow, (i and $\mathrm{j}$ ): Between attachment sites of a day 24 pregnant sow, ( $\mathrm{k}$ and 1$)$ : At attachment sites of a day 24 pregnant sow and ( $m$ and $n$ ): Negative controls for localization $(\mathrm{x} 400)$

Huppertz et al., 1998; Bjorn et al., 2000; Salamonsen, 1999; Qin et al., 2003). MMPs are proteolytic enzymes that depend on zinc and calcium ions and are the main degradation factors for the ECM in various tissues (Nagase and Woessner, 1999) In non-invasive placenta species, our knowledge of the roles of MMPs is limited. In the study, MMP-2 protein expression at attachment sites was higher than between attachment sites during implantation in sows which suggests that remodeling of endometrium at attachment sites is greater than between 
attachment sites. Immunohistochemical localization of MMP-2 and -9 has been observed in luminal epithelium and stroma which suggests that these genes play important role in the cell to cell contact and remodeling of endometrial epithelia and stoma during the implantation period in sows. The immunostaining of MMP-2 and -9 in stroma at attachment sites was stronger than between attachment sites during implantation. ECM including collagen type-I, -IV, laminin and fibronectin expression declined around the start of implantation both spatially and temporally (Yamada et al., 2002a, b). This is compatible with findings from rodents, humans and even bovines with a non-invasive placenta (Wang et al., 2004; Xu et al., 2000; Das et al., 1997). Therefore, MMP-2 and -9 may play significant regulatory roles for gelatin and collagen type IV during implantation, even in sows. These findings demonstrate that MMP-2 and -9 may mediate ECM degradation which is similar to cows (Maj and Kankofer, 1997; Das et al., 1997; Patel et al., 2004).

During implantation, MMPs are believed to play roles in the tissue remodelling that accompanies decidualization in the endometrium and in embryo invasion. Johnson et al. (2003) and Lin et al. (2007) reported a stromal decidualization like response in the pregnant ovine and porcine uterus by studying osteopontin, integrin $\alpha \mathrm{V}$ and $\beta 3$ expression. Porcine embryos do not invade the uterine wall. However MMP-2 and -9 are expressed in stroma and increases at attachment sites compared with between attachment sites during implantation.

The phenomenon indicates the important role of MMP-2 and -9 in conceptus survival, since stroma is crucial for maintaining morphogenesis, hormonal responsiveness and secretory function of the uterine epithelium. Moreover, epithelial-stromal interactions have been implicated in development, growth, differentiation and adult function of the uterus. So there may be a decidualization-like response in pregnant porcine uterus stroma, though the degree is lower.

Embryos of all mammals are inherently invasive and can attach to and invade a diverse array of artificial ectopic sites and biological matrices without discrimination or need for hormonal priming. However, the luminal epithelium is unique in that it serves as a barrier to conceptus invasion until it is cyclically transformed to a receptive state that responds to embryonic signals and permits adhesive contact with conceptus epithelium (Denker, 1993). In the study, immunostaining for MMP-2 and -9 in luminal epithelium was observed during implantation and the mechanism of this type of regulation remains to be established.

\section{CONCLUSION}

Results from this study provide evidence that gelatinase is an important factor during embryo successful implantation in sows. MMP-2 mRNA and protein expression during implantation coin-cides with ECM degradation in sows. To explore the implantation mechanism, it can focus on function of MMP-2 and -9 in endometrial stroma and luminal epithelium, since pig is the only species that demonstrates true epitheliochorial placental animal.

\section{ACKNOWLEDGEMENTS}

The researchers greatly appreciate Beijing Huadu Swine Breeding Company, LTD for collecting samples. This study was supported by National High-Technology Research Development Program of China (No. 007AA10Z166and2008AA101008) and National Scientific and Technical Supporting Programs of China (No. 2007BAD71B04).

\section{REFERENCES}

Bai, S.X., Y.L. Wang, L. Qin, Z.J. Xiao, R. Herva and Y.S. Piao, 2005. Dynamic expression of matrix metalloproteinases (MMP-2, -9 and -14) and the tissue inhibitors of MMPs (TIMP-1, -2 and -3 ) at the implantation site during tubal pregnancy. Reproduction, 129: 103-113.

Bjom, S.F., N. Hastrup, J.F. Larsen, L.R. Lund and C. Pyke, 2000. Messenger RNA for membrane-type 2 matrix metalloproteinase, MT2-MMP, is expressed in human placenta of first trimester. Placenta, 21: $170-176$.

Blankenship, T.N. and B.F. King, 1994. Identification of 72-kilodalton type IV collagenase at sites of trophoblastic invasion of macaque spiral arteries. Placenta, 15: 177-187.

Burghardt, R.C., G.A. Johnson, L.A. Jaeger, H. Ka, J.E. Garlow, T.E. Spencer and F.W. Bazer, 2002. Integrins and extracellular matrix proteins at the maternal-fetal interface in domestic animals. Cells Tissues Organs, 172: 202-217.

Das, S.K., S. Yano, J. Wang, D.R. Edwards, H. Nagase and S.K. Dey, 1997. Expression of matrix metalloproteinases and tissue inhibitors of metalloproteinases in the mouse uterus during the peri-implantation period. Dev. Genet., 21: 44-54.

Denker, H.W., 1993. Implantation: A cell biological paradox. J. Exp. Zool., 266: 541-558. 
Huang, M.C., H.Y. Chen, H.C. Huang, J. Huang and J.T. Liang et al., 2006. C2GnT-M is downregulated in colorectal cancer and its re-expression causes growth inhibition of colon cancer cells. Oncogene, 25: 3267-3276.

Huppertz, B., S. Kertschanska, A. Y. Demir, H.G. Frank and P. Kaufmann, 1998. Immunohistochemistry of matrix metalloproteinases (MMP), their substrates and their inhibitors (TIMP) during trophoblast invasion in the human placenta. Cell Tissue Res., 291: 133-148.

Johnson, G.A., R.C. Burghardt, M.M. Joyce, T.E. Spencer, F.W. Bazer, C. Pfarrer and C.A. Gray, 2003. Osteopontin expression in uterine stroma indicates a decidualization-like differentiation during ovine pregnancy. Biol. Reprod., 68: 1951-1958.

Kizaki, K., K. Ushizawa, T. Takahashi, O. Yamada and J. Todoroki et al., 2008. Gelatinase (MMP-2 and -9) expression profiles during gestation in the bovine endometrium. Reprod. Biol. Endocrinol., 6: 66-66.

Kizaki, K., O. Yamada, H. Nakano, T. Takahashi, N. Yamauchi, K. Imai and K. Hashizume, 2003. Cloning and localization of heparanase in bovine placenta. Placenta, 24: 424-430.

Kyriazakis, I. and C.T. Whittemore, 2006. Reproduction, Whittemores Science and Practice of Pig Production. 3rd Edn., Oxford: Blackwell Publishing, UK., ISBN: 978-1-4051-2448-5, pp: 105-147.

Lin, H.C., X. Wang, G.F. Liu, J.L. Fu and A.G. Wang, 2007. Expression of alphaV and beta3 integrin subunits during implantation in pig. Mol. Reprod. Dev., 74: 1379-1385.

Livak, K.J. and T.D. Schmittgen, 2001. Analys is of relative gene expression data using real-time quantitative PCR and the 2(-Delta Delta C (T)) method. Methods, 25: 402-408.

Lord, E., B.D. Murphy, J.A. Desmarais, S. Ledoux, D. Beaudry and M.F. Palin, 2006. Modulation of peroxisome proliferator-activated receptor delta and gamma transcripts in swine endometrial tissue during early gestation. Reproduction, 131: 929-942.

Maj, J.G. and M. Kankofer, 1997. Activity of 72-kDa and 92-kDa matrix metalloproteinases in placental tissues of cows with and without retained fetal membranes. Placenta, 18: 683-687.

Nagase, H. and J.F.J. Woessner, 1999. Matrix metalloproteinases. J. Biol. Chem., 274: 21491-21 494.

Patel, O.V., O. Yamada, K. Kizaki, T. Takahashi, K. Imai and K. Hashizume, 2004. Quantitative analysis throughout pregnancy of placentomal and interplacentomal expression of pregnancy-associated glycoproteins-1 and -9 in the cow. Mol. Reprod. Dev., 67: 257-263.
Qin, L., Y.L. Wang, S.X. Bai, S.H. Ji, W. Qiu, S. Tang and Y.S. Piao, 2003. Temporal and spatial expression of integrins and their extracellular matrix ligands at the maternal-fetal interface in the rhesus monkey during pregnancy. Biol. Reprod., 69: 563-571.

Rechtman, M.P., J. Zhang and L.A. Salamonsen, 1999. Effect of inhibition of matrix metalloproteinases on endometrial decidualization and implantation in mated rats. J. Reprod. Fertil., 117: 169-177.

Salamonsen, L.A., 1999. Role of proteases in implantation. Rev. Reprod., 4: 11-22.

Schmittgen, T.D. andK.J. Livak, 2008. Analyzing real-time PCR data by the comparative C(T) method. Nat. Protoc., 3: 1101-1108.

Takagi, M., D. Yamamoto, M. Ohtani and A. Miyamoto, 2007. Quantitative analysis of messenger RNA expression of matrix metalloproteinases (MMP-2 and MMP-9), tissue inhibitor-2 of matrix metalloproteinases (TIMP-2) and steroidogenic enzymes in bovine placentomes during gestation and postpartum. Mol. Reprod. Dev., 74: 801-807.

Uekita, T., K. Yamanouchi, H. Sato, H. Tojo, M. Seiki and C. Tachi, 2004. Expression and localization of matrix metalloproteinases (MT1-MMP, MMP-2) and tissue inhibitor of metalloproteinase-2 (TIMP-2) during synepitheliochorial placentation of goats (Capra hircus). Placenta, 25: 810-819.

Wang, H.M., X. Zhang, D. Qian, H.Y. Lin and Q.L. Li et al., 2004. Effect of ubiquitin-proteasome pathway on mouse blastocyst implantation and expression of matrix metalloproteinases-2 and -9. Biol. Reprod., 70: 481-487.

Wooding, F.B., 1992. Current topic: The synepitheliochorial placenta of ruminants: Binucleate cell fusions and hormone production. Placenta, 13: 101-113.

Xu, P., Y.L. Wang, S.J. Zhu, S.Y. Luo, Y.S. Piao and L.Z. Zhuang, 2000. Expression of matrix metalloproteinase $-2,-9$ and -14 , tissue inhibitors of metalloproteinase- 1 and matrix proteins in human placenta during the first trimester. Biol. Reprod., 62: 988-994.

Yamada, O., J. Todoroki, K. Kizaki, T. Takahashi and K. Imai et al., 2002a. Expression of prolactin-related protein $\mathrm{I}$ at the fetomaternal interface during the implantation period in cows. Reproduction, 124: $427-437$.

Yamada, O., J. Todoroki, T. Takahashi and K. Hashizume, $2002 \mathrm{~b}$. The dynamic expression of extracellular matrix in the bovine endometrium at implantation. J. Vet. Med. Sci., 64: 207-214. 